\title{
Oral Appliance as a Sensory Trick to Manage Tardive Dyskinesia of Tongue: A Case Report
}

\author{
Jun-Hee Shin, Mee-Eun Kim, Hye-Kyoung Kim \\ Department of Oral Medicine, College of Dentistry, Dankook University, Cheonan, Korea
}

Received May 12, 2020

Revised May 28, 2020

Accepted May 28, 2020

\section{Correspondence to:}

Hye-Kyoung Kim

Department of Oral Medicine, College of Dentistry, Dankook University, 119 Dandaero, Dongnam-gu, Cheonan 31116, Korea

Tel: +82-41-550-1913

Fax: +82-505-434-7951

E-mail: 12170144@dankook.ac.kr

https://orcid.org/0000-0002-0734-5533
Tardive dyskinesia (TD) is continuous, repetitive movement disorder of tongue, lip or jaw, induced by medication. It causes pain and dysfunction of oral structures but also interferes with dental treatment and overall social life of patients. As a dentist, it is imperative to recognize and manage TD, although currently, there is no definitive treatment for TD. This article reports a patient with TD of tongue, successfully managed with an oral appliance mimicking sensory tricks. Considering the limited treatment options for TD, an oral appliance, a simple and conservative approach, can be a meaningful treatment for some patients with orofacial dyskinesia.

Key Words: Geste antagoniste; Oral appliance; Sensory trick; Tardive dyskinesia

\section{INTRODUCTION}

Tardive dyskinesia (TD) is involuntary, repetitive movement disorder of the tongue, lip or jaw, induced by medication [1]. About 15\%-30\% of patients, who receive longterm medication, develop tardive TD [1]. The exact mechanisms of adverse medical reactions that cause TD are not well defined. Several hypotheses are suggestive of abnormalities in dopaminergic, gamma-aminobutyric acid-releasing (GABAergic), cholinergic pathways leading to excessive movements [1-3]. General treatment strategies are medication, botulinum toxin injection and surgical intervention, but their effects are controversial. Some patients partially control the dyskinetic movements with the use of tactile stimulation known as sensory tricks or 'geste antagoniste' [1]. Dentists should recognize and manage orofacial dyskinesia not only because it causes pain and dysfunction of oral structures but also because it severely interferes with dental treatment and social life of patients. In this report, a patient with TD of tongue is introduced, who was prescribed a mandibular appliance mimicking his sensory trick.

\section{CASE REPORT}

A 56-year old male presented with a chief complaint of involuntary tongue movement and speech difficulty in July 1st, 2019. According to the patient, his symptom started after taking antidepressant drugs for tinnitus. The lingual movement continued even after the withdrawal of the medication in January 2019.

His past medical history revealed long-term use of medication. He had been taking ropinirole $2 \mathrm{mg}$ (Ropimax; Korean Drug Co., Ltd., Seoul, Korea), alprazolam 0.25 mg (Xyren; Kwangdong Pharm Co., Ltd., Seoul, Korea), propranolol 40 mg (Indenol; Dongkwang Pharm Co., Ltd., Seoul, Korea) twice a day for 8 years for restless leg syndrome and anxiety disorder. During the last 2 years, venlafaxine $150 \mathrm{mg}$ (Venexor; Kolon Pharm Co., Ltd., Gwacheon, Korea), amitriptyline $10 \mathrm{mg}$ (Enafon; Whanin Pharm Co., Ltd., Seoul, Korea) were additionally prescribed for tinnitus. Gradually, the lingual dyskinesia started to develop. In January 2019, the additional drugs were discontinued to stop the tongue movement, but with no benefit.

Copyright (c) 2020 Korean Academy of Orofacial Pain and Oral Medicine. All rights reserved.

(c) This is an open-access article distributed under the terms of the Creative Commons Attribution Non-Commercial License (http://creativecommons.org/licenses/by-nc/4.0/), which permits unrestricted non-commercial use, distribution, and reproduction in any medium, provided the original work is properly cited. 
Extraoral examination showed continuous side to side movement of his tongue and mandible. He had mild tenderness on his bilateral masseter and temporalis upon palpation. His mouth opening was normal with $50 \mathrm{~mm}$ of comfortable range of motion and mild click on the right side. Panoramic image revealed no pathologic findings in bony structures (Fig. 1).

Intraoral examination revealed several ulcers on the dorsal surface of his tongue and right buccal mucosa (Fig. 2). Mucosal surface was dry and the salivary flow rate was low (unstimulated: $0.04 \mathrm{~mL} / \mathrm{min}$, stimulated: $0.3 \mathrm{~mL} / \mathrm{min}$ ). For closer examination of the tongue, a mirror was put inside the mouth. At the exact moment, the restless tongue movement stopped.

\section{Diagnosis and treatment}

From the patient's medical history and clinical characteristics, he was diagnosed with drug-induced TD and druginduced hyposalivation. Considering the prominent characteristics of geste antagoniste, a mandibular soft appliance was fabricated to reduce the tongue movement and to protect oral mucosa (Fig. 3). When the patient put on the appliance, the dyskinetic tongue movement immediately

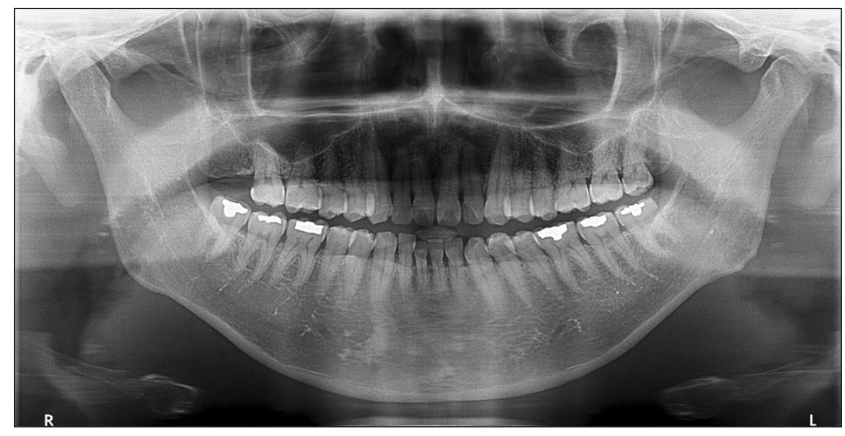

Fig. 1. Panoramic image at initial visit. subsided (Fig. 4). To prevent possible occlusal changes, he was instructed to use the appliance during the day except mealtime. Patient's well-balanced occlusal contacts were carefully checked with occlusal papers on every recall.

At 2 weeks follow-up, the tongue movement was well managed with the appliance. Soreness of bilateral masseter and temporalis muscles was resolved. Intraoral ulcers were completely healed (Fig. 5). The dryness of intraoral mucosa was improved. During fortnightly observation for 8 months, the patient reported overall reduction of the involuntary tongue movement and traumatic events on orofacial structures. He was able to fix his cervical abrasions on his maxillary teeth, which were left untreated due to his dyskinesia. At the last appointment, he stopped his anxiety medicine with the improvement of his psychosocial status.

\section{DISCUSSION}

According to the American psychiatric association, TD

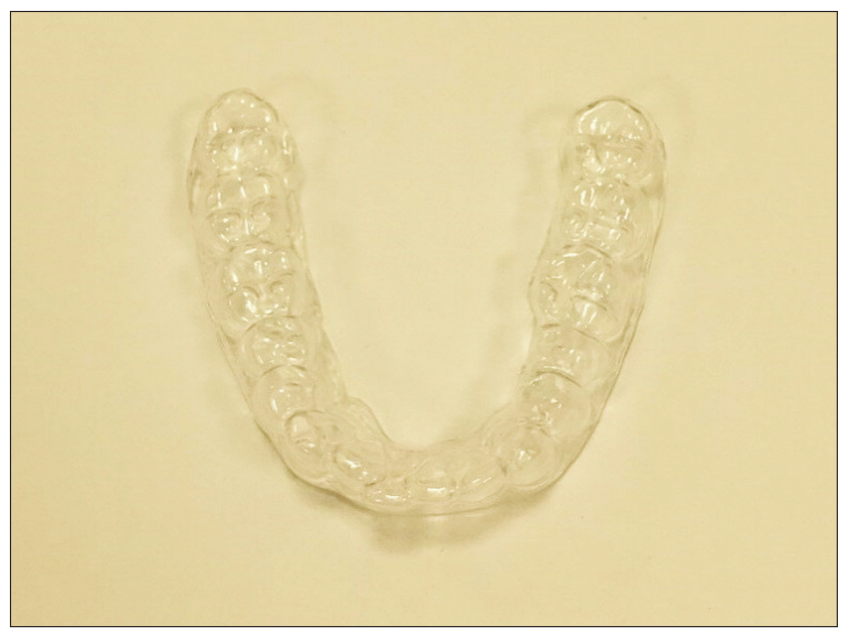

Fig. 3. Oral appliance used to manage lingual dyskinesia (Bioplast 1 mm; Scheu Dental GmbH, Iserlohn, Germany).
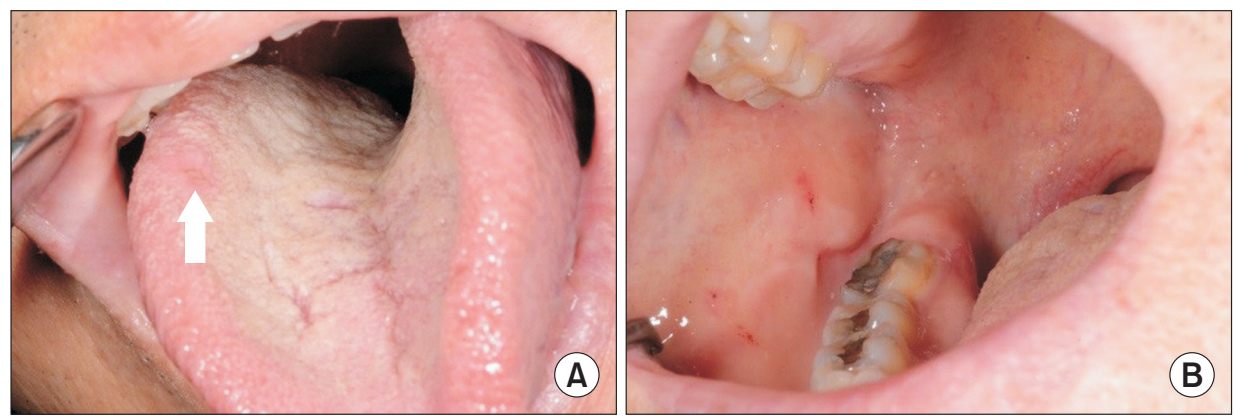

Fig. 2. Intraoral pictures at initial visit. (A) Traumatic ulcer on the right dorsal surface of the tongue (white arrow). (B) Several traumatic petechiae of the right buccal mucosa. 
is characterized by repetitive, involuntary, and stereotyped motor disorder associated with antipsychotic agents [4]. There are multiple hypotheses to explain the disease process, including dopamine D2 receptor supersensitivity, gamma-aminobutyric acid (GABA) insufficiency, maladaptive synaptic plasticity, striatal neurodegeneration and enhanced serotonin 5-hydroxytryptamine2 receptors [5]. Antipsychotic drugs are known to cause of TD, but recent reports have suspected other wide variety of medications, such as anti-Parkinson's agents, antidepressants and anticholinergics [3]. Diagnosis of TD is based on clinical observation and patient history [1]. In this case, the patient had been taking ropinirole (dopamine D agonist) for 8 years and amitriptyline (tricyclic antidepressant) and venlafaxine (serotonin noradrenaline reuptake inhibitor) for the last 2 years, until the additional drugs were stopped by his

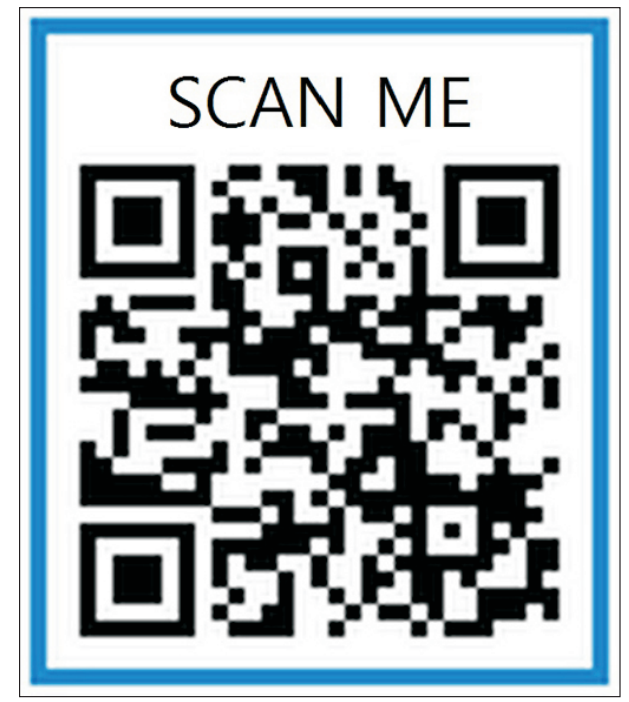

Fig. 4. QR code for the movie showing the effect of oral appliance on dyskinesia of the tongue. neurologist due to the lingual dyskinesia. These medications, regulating multiple neurotransmitters, are suspected to have caused TD of the tongue [2,3].

During the intraoral examination of the patient, the dyskinetic movement of the tongue was controlled by soft touch of the mirror. This brought insight to use an oral appliance as a sensory trick. The exact mechanism of sensory trick remains elusive, but several imaging studies including cervical dystonia, blepharospasm, writer's cramp have illustrated its effect on multiple brain centers [6-8]. A theoretical explanation is the compensatory mechanism during the early course of the disease by sensory overstimulation [9]. According to Okeson [10], when an occlusal appliance is placed between the teeth, it provides a change in peripheral sensory input often resulting in a decrease in central nervous system-induced parafunction.

Botulinum toxin is considered as the first-line therapy for focal dystonia [1]; however, tongue is the most difficult organ in the stomatognathic system to target with botulinum toxin injection. It has crucial functions in speech, deglutition, mastication, and respiration. Paralyzing the tongue muscles may bring dangerous consequences, such as dysphagia, aspiration pneumonia, and even dyspnea $[11,12]$.

In comparison, the oral appliance in this article is simple, conservative and easy to follow. Okeson [10] illustrates three factors for a successful oral appliance; 1. type selection, 2. fabrication and adjustment, 3. patient cooperation. First, a soft appliance was selected to protect oral structures from trauma by TD [10]. A thin transparent material, Bioplast (1 mm; Scheu Dental GmbH, Iserlohn, Germany), was used so that the patient could wear it comfortably during the day. Second, the appliance was fabricated and adjusted on mandibular teeth because the patient's tongue mostly touched
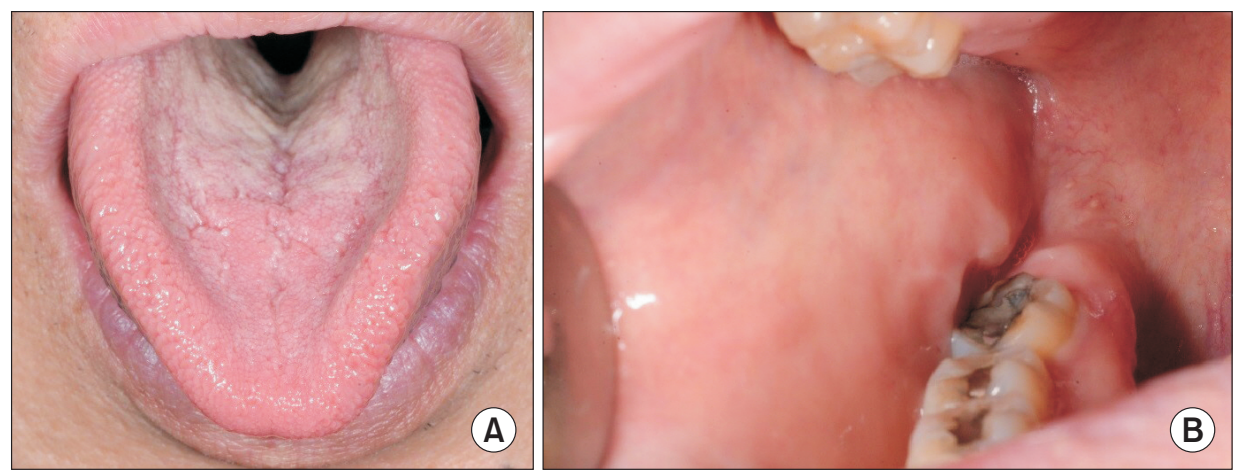

Fig. 5. Intraoral pictures at 2 weeks recall from the first visit. (A) Healing of the dorsal surface of the tongue. (B) Healing of the right buccal mucosa. 
the lower teeth at resting position. Although most oromandibular disorders are task-specific [13], the patient's lingual dyskinesia appeared at rest and reduced with tactile stimulation. Lastly, the patient well-cooperated on wearing the appliance because the soft and thin appliance comfortably adjusted to patient's oral structure. Since all three factors from Okeson were satisfied, the appliance was able to maximize its benefit on the patient.

Although this case demonstrates dramatic effects of the oral appliance, there are several limitations. First, it is a single case report and careful case selection to maximize the benefit of the oral appliance is necessary. According to Kazuya's report of 128 oromandibular dystonia cases, patients with jaw closing dystonia, and intraoral sensory tricks showed favorable response to oral appliances [13]. Among the responders, subjective relief of symptoms was average $30 \%$, which implies limited effectiveness of the splint $[13,14]$. In a study by Kägi et al. [9], significant improvement with oral appliance was observed in patients with dystonia of lower severity, shorter disease-duration, and lower visuotactile discrimination thresholds. With careful case selection based on above mentioned factors, oral appliance can be a meaningful treatment option for certain patients.

Second, this case is an 8-month report, which needs further observation to verify the effectiveness of the oral appliance. For 8 months follow-ups, the patient's dyskinesia seemed to be well managed with the appliance. According to Okeson [10], patients with parafunction who had positive symptom relief early in wearing the appliance may have symptoms return with continued use because of the concept of sensory adaptation. Placebo effect is also possible with any treatment and whether the symptom subsided due to the actual therapeutic effect or regressed to the mean needs further evaluation [10]. Long-term follow-ups are necessary to draw a definitive conclusion about the effects of the oral appliance.

In conclusion, considering the limited treatment options for TD, an oral appliance, mimicking sensory trick, is a meaningful option for certain patients. The concept of 'geste antagoniste' may not be successful in all cases; however, with careful case selection, the easy to follow appliance not only provides partial relief of symptoms but also goes further in improving the quality of life for some patients.

\section{CONFLICT OF INTEREST}

No potential conflict of interest relevant to this article was reported.

\section{ORCID}

\author{
Jun-Hee Shin \\ https://orcid.org/0000-0002-8306-1460 \\ Mee-Eun Kim \\ https://orcid.org/0000-0001-9332-532X \\ Hye-Kyoung Kim \\ https://orcid.org/0000-0002-0734-5533
}

\section{REFERENCES}

1. Clark GT, Ram S. Orofacial movement disorders. Oral Maxillofac Surg Clin North Am 2016;28:397-407.

2. Chen JM, Yan Y. Long-term follow-up of a patient with venlafaxine-induced diurnal bruxism treated with an occlusal splint: a case report. World J Clin Cases 2019;7:516-524.

3. Cornett EM, Novitch M, Kaye AD, Kata V, Kaye AM. Medicationinduced tardive dyskinesia: a review and update. Ochsner J 2017;17:162-174

4. Caligiuri MP. Tardive dyskinesia: a task force report of the American Psychiatric Association, Washington, D.C. Psychiatr Serv 1993;44.

5. Farag AM, Mier RW, Correa LP. Utilizing the concept of geste antagoniste for conservative management of oro-mandibular tardive dyskinesia: a case report and mini-review. Cranio 2016;34:338-342.

6. Naumann M, Magyar-Lehmann S, Reiners K, Erbguth F, Leenders KL. Sensory tricks in cervical dystonia: perceptual dysbalance of parietal cortex modulates frontal motor programming. Ann Neurol 2000;47:322-328.

7. Gómez-Wong E, Martí MJ, Cossu G, Fabregat N, Tolosa ES, VallsSolé J. The 'geste antagonistique' induces transient modulation of the blink reflex in human patients with blepharospasm. Neurosci Lett 1998;251:125-128.

8. Peller M, Zeuner KE, Munchau A, et al. The basal ganglia are hyperactive during the discrimination of tactile stimuli in writer's cramp. Brain 2006;129(Pt 10):2697-2708.

9. Kägi G, Katschnig P, Fiorio M, et al. Sensory tricks in primary cervical dystonia depend on visuotactile temporal discrimination. Mov Disord 2013;28:356-361.

10. Okeson JP. Management of temporomandibular disorders and occlusion. 8th ed. St. Louis: Elsevier; 2020. pp. 385-407.

11. Blitzer A, Brin MF, Fahn S. Botulinum toxin injections for lingual dystonia. Laryngoscope 1991;101(7 Pt 1):799.

12. Yoshida K. Botulinum neurotoxin therapy for lingual dystonia using an individualized injection method based on clinical fea- 
tures. Toxins (Basel) 2019;11:51.

13. Yoshida K. Sensory trick splint as a multimodal therapy for oromandibular dystonia. J Prosthodont Res 2018;62:239-244.
14. Lo SE, Gelb M, Frucht SJ. Geste antagonistes in idiopathic lower cranial dystonia. Mov Disord 2007;22:1012-1017. 